\title{
Using Process Mining to Support Theorizing About Change in Organizations
}

\author{
Thomas Grisold \\ University of \\ Liechtenstein \\ thomas.grisold@uni.li
}

\author{
Bastian Wurm \\ Vienna University of \\ Economics and Business \\ bastian.wurm@wu.ac.at
}

\author{
Jan Mendling \\ Vienna University of \\ Economics and Business \\ jan.mendling@wu.ac.at
}

\author{
Jan vom Brocke \\ University of \\ Liechtenstein \\ jan.vom.brocke@uni.li
}

\begin{abstract}
Process mining refers to a family of algorithms used to computationally reconstruct, analyze and visualize business processes through event log data. While process mining is commonly associated with the improvement of business processes, we argue that it can support theorizing about change in organizations. Central to our argument is that process mining algorithms can support inductive as well as deductive theorizing. Process mining algorithms can extend established theorizing in a number of ways and with respect to different research agendas and phenomena. We illustrate our argument in relation to two types of change; endogenous change that evolves over time and exogenous change that follows a purposeful intervention. Drawing on the discourse of routine dynamics, we propose how different process mining features can reveal insights about the dynamics of organizational routines.
\end{abstract}

\section{Introduction}

Organizational change is a complex and dynamic phenomenon. It unfolds over time and is affected by various contextual factors [1]. It can happen intentionally when organizations embark on change initiatives, and it can happen unintentionally over time. To capture and explain these dynamics, research has approached change from a process-theoretical view [2].

Process studies aim to explain temporally evolving phenomena [3]. In contrast to variance studies, which reveal statistical correlations between antecedents, factors and outcomes of change, process theories aim to answer how and why change occurs [4]. They account for the specific actions, activities and choices that are made by actors at specific points in time, and they identify the contingencies and generative mechanisms that underlie processes of change [1].

Organizational work is increasingly supported by information systems. Digital technology is imbricated with human practices and embedded in organizational routines [5]. When actors use these technologies, they leave digital traces. Recent claims suggest that research can draw on these data in order to reconstruct and analyze phenomena in terms of the specific activities that are performed at specific points in time $[6,7]$. As seen from this perspective, digital trace data can capture "reality in flight" [8] and support process theorizing [9].

In this article, we discuss the use of process mining as a computational method to support theorizing about change in organizations. Process mining refers to a family of algorithms that use event logs from information systems (e.g. ERP systems) to reconstruct and analyze business processes [10]. While process mining is commonly used to monitor and improve business processes, we argue that it can reveal important insights about how process work is carried out and changes over time [11]. In this article, we pursue the following research question:

How and to what extent can process mining support theorizing about change in organizations?

Building on recent claims that process mining can contribute to organizational process research [11], we aim to present a wider spectrum of algorithms and relate them to different stages of the research cycle. Central to our argument is that process mining algorithms can support both inductive and deductive theorizing, and thus, they can augment process theorizing in a number ways [6]. In particular, since digital trace data record a wide spectrum of actions performed by the use of information systems, process mining offers a more fine-granular view of work activities. Drawing on two types of change processes -endogenous change that evolves over time and exogenous change that follows punctuated interventions-, we illustrate how the use of process mining algorithms can lead to different insights about change in organizational routines.

We proceed as follows. First, we discuss process research as the theoretical background of our work. Second, we introduce process mining and explain how process mining algorithms can support inductive 
and deductive theorizing. Drawing on organizational routines research, we then illustrate how process mining can be used to explore change dynamics in organizational routines. We conclude by pointing to opportunities for future research.

\section{Theoretical Background}

\subsection{Organizational Change and Process Theorizing}

Organizational change, broadly defined as an organization's transition from one state to another, has been approached from different angles and with respect to different phenomena $[1,4]$.

Process studies explore patterns and dynamics that occur within change processes. They aim to explain how and why change unfolds over time [4]. In doing so, they explain change in terms of the underlying process(es), i.e. the constituting events, activities and decisions. Process studies prioritize "activity over product, change over persistence, novelty over continuity, and expression over determination" [12, p.2]. On this view, organizations (and their structures) are stable and persistent but at the same time, they are continuously constructed and reconstructed and hence, always changing. To understand change from a process perspective, attention is directed to temporality and the question of what is done, when it is done and in what context [3].

While organizational change is defined and conceptualized in different ways [2], we are focusing on two types of change that are often discussed in the literature; endogenous change that evolves from within the organization, and exogenous change that follows purposeful interventions $[13,14]$.

Endogenous evolutionary change is characterized by different change patterns that emerge from within the organization. Van de Ven and Poole [2] point out that small patterns can form larger patterns of change. Change is endogenous in the sense that it unfolds without an explicit agenda or external interventions. For example, from a process perspective, organizational failure can result from small erroneous actions that cause major problems when they are repeated over time [15]. In a similar vein, organizational routines researchers identified different mechanisms leading to generative changes in action patterns [16].

Exogenous punctuated change is tied to specific interventions that, after being implemented at $t_{0}$, aim to result in a new state at $t_{1}$. Such interventions can interfere with existing ways of organizing and often lead to unexpected consequences. There are different types of exogenous change. For example, research examined how intended changes in institutional logics are mirrored in actions, thoughts and emotions of organizational actors [17]. Exogenous change can also be triggered by the implementation of artifacts which necessitate new interaction patterns. This can affect existing forms of organizing in considerable ways [5].

While there are several strategies to theorize from process data [9], it is important to identify "critical events" that mark patterns of change dynamics and indicate how multiple temporalities, levels of analysis and contextual factors together constitute a phenomenon $[1,4]$. Process studies are commonly based on qualitative data, encompassing observations, interviews and other data sources (e.g. archival data). The majority of process studies is inductive but they can also be deductive to test hypothesized models of change $[4,18]$.

Following recent claims, process research may also capitalize on the increasing use of digital technologies $[19,11]$. This might prove useful in two respects. First, actors leave digital traces when they use digital technologies and these traces can support researchers in reconstructing and analyzing work sequences [7, 20]. Second, in light of increasingly sophisticated algorithms, large amounts of data can be analyzed in different ways [6].

\subsection{Computationally-Driven Theorizing}

Information technology increasingly penetrates social and organizational life [21], and actors leave digital traces when they use digital technologies. IT systems store information about actions, actors and times of executions in event logs. While digital traces are naturally occurring data [22] not explicitly designed for research purposes, they can reveal how events, when aligned in a sequential order, constitute a phenomenon $[8,19]$. More and more scholars make an argument for using computationally-driven theorizing to analyze digital trace data [6, 7, 23, 24]. For example, research can use social media data to analyze social networks, or email messages to analyze communication patterns.

It has been suggested that computational methods from the field of business process management, i.e. process mining, can contribute to organizational process research [11]. Analyzing digital trace data as "records of actions" [7] through process mining has two central implications. On the one hand, it extracts temporal relations among events from an event $\log$ and aligns them in sequences. On the other hand, it can discover processes, analyze them over time and test their conformance with respect to predefined models. These features can support the research cycle both in terms of induction as well as deduction. In the following, 
we will present process mining as a method to support theorizing about change in organizations. We highlight that computationally supported theory development needs to be contextualized and complemented with human sense making [6].

\section{Conceptualizing Process Mining as a Method to Theorize about Change in Organizations}

Process mining encompasses a family of algorithms in order to extract, visualize, and analyze processual information from event logs [10]. In practice, process mining has emerged as a powerful tool to support process improvement [25].

The three most fundamental and comprehensive process mining techniques are: (1) Process Discovery, (2) Conformance Checking, and (3) Enhancement [10]. (1) Process Discovery algorithms mine an event log and extract descriptive information about how the process is performed. A process model is generated, while no a-priori information about the process is required [10]. (2) Conformance Checking is used to compare the process as captured by the event log with a prescriptive model of that process [10]. There are various measures to determine the extent to which a process model corresponds with the actual process (as captured by the event log). Algorithms for process (3) Enhancement can be used to improve the process by detecting bottlenecks or by further analyzing a mismatch between a process model and the actual process execution [26]. While most process mining algorithms focus on the sequence of activities, more and more algorithms are developed dealing with other elements of a process, such as resources, case characteristics, and changes over time [27].

\subsection{Using Process Mining for Theory-Building and Theory-Testing}

In the following, we extend previous claims to use process mining in process research $[11,28]$ and discuss how different process mining algorithms can contribute to different stages of the research cycle. We argue that process mining can support inductive and deductive theorizing about change in organizations. Process mining draws on event logs containing a large number of data records that are collected over a number of repetitions. These logs also contain actions that occur not very often, which means that the use of process mining can give a detailed picture of how the process is or was carried out. Furthermore, since digital trace data reflect actions taken by the use of information systems, they might not be readily accessible with traditional data

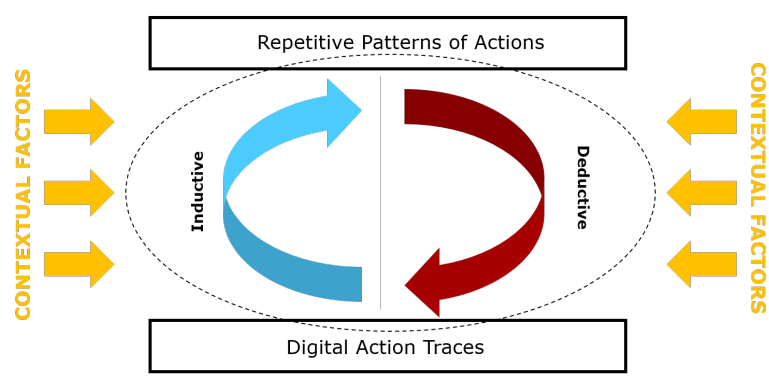

Figure 1. Inductive and Deductive Theorizing with Process Mining

collection approaches (e.g. interviews or observations). Therefore, the use of process mining may complement existing approaches by providing a more fine-granular view of organizational work.

Figure 1 illustrates how process mining can support inductive and deductive theorizing by iterating between action traces from event logs and derived patterns of actions. It is important to note that some algorithms are neither purely inductive or deductive. To highlight this fact, we show how process discovery algorithms can be used for both, inductive and deductive theorizing.

Process Mining to Support Inductive Theorizing. Process mining algorithms can support inductive theorizing by revealing patterns in the data that can be used for theory development. These algorithms are employed in an explorative manner with very limited ex-ante theoretical framing and information about how the process is or should be executed. By processing event logs including records of actions which were taken within work sequences, process mining can reveal how organizational work is carried out. Since event logs contain numerous instances of a specific work process, process mining can provide more abstract views (how the process is carried out in most of the cases) as well as more fine-granular views (how the process is enacted in less frequent cases). By visualizing process work, one can detect anomalies or surprising events, e.g. when deviations occur. With reference to Berente et al.'s [6] distinction between synchronic and diachronic analysis in computationally-intensive theorizing, process mining can be primarily used for diachronic analysis by aligning patterns along with temporal relationships (e.g. by using the visualized process as a temporal structure) [11]. However, it might also support synchronic analysis (e.g. in terms of steps frequently carried out or anomalies). In both cases, additional data needs to be collected. We report on two exemplary algorithms, Process Discovery and Variant Detection, and we explain how researchers can employ them to support inductive theorizing.

Process Discovery generates a process model from 
an event log. The resulting model visualizes how the process is carried out, and different paths and their respective frequencies can be explored. The most basic algorithm to do this is the alpha-algorithm [27], however, more sophisticated algorithms exist [29]. An example for the use of process discovery is Lindberg et al.'s study [30] of an open-source software community, where the authors mine trace data to understand how developers resolve code interdependencies [6]. Subsequently, the authors explore the data by means of qualitative research and they compute various statistical measures to understand how the resolution process takes place.

Another suitable class of algorithms to derive patterns of actions is Variant Detection. Hompes and others use a Markov cluster algorithm to derive clusters of process variants [31]. For example, process performances in hospitals largely depend on, and hence can be clustered according to, attributes of the treated cases, e.g. age and diagnosis [31]. As this class of algorithms reveals different process sequences and how they are performed, it informs the researcher about the teleology and dialectic [2] of a process. Thus, it is possible to identify different process enactments with respect to different goals that are pursued.

Process Mining to Support Deductive Theorizing. Process mining algorithms can be employed deductively to predict and test hypotheses or propositions. Deductive theorizing can strengthen existing theories about how work processes are carried out with respect to different factors, e.g. temporal patterns or the implementation of new regulations [4, 18]. Process mining can be used to test assumptions across organizations but it can also be used within one and the same case after inductive theorizing has been conducted. We present exemplary algorithms: Concept Drift, Conformance Checking and Process Discovery. We deliberately choose to present Process Discovery once more in order to demonstrate that the same class of algorithms may be used for theory-building and/or theory-testing.

Concept Drift algorithms detect changes in the execution of a process. This involves finding the point in time when a change takes place, identifying the type of change, and reconstructing how the process developed [32]. A common technique used for detecting concept drift is the sliding windows approach [33] where the log-file is divided into two consecutive sub-logs, so-called windows. Windows are then compared and tested in terms of statistical differences. If there is significant variation in the log-file, a drift is indicated. The type of drift can vary with regards to the speed of change and its permanence [32]. Concept drift algorithms can be employed to correlate hypothesized change and actual change, as recorded by the event log. When drift takes place, the researcher can further explore how it unfolds, validate assumptions or revise working hypotheses.

Hypothesized changes of a process can be specified in process models. Conformance Checking algorithms can compare such hypothesized models with traces collected in event logs. Behavioral measures allow for subsequent reflection to determine the extent to which hypothesized and actual process behavior overlap [34]. Process Discovery can be used ex-post to validate research findings and check whether the developed theory suits the data. This stands in contrast to the inductive use of process discovery where the explorative aspect is paramount.

\subsection{Contextualization of Process Mining Data}

Since computational theorizing cannot replace human theorizing [9], the use of process mining needs to be complemented with human imagination, sense-making and creativity [6]. The sole use of process mining cannot provide enough information to explain how or why change occurs, as it does not reflect actors' intentions, reasoning, or feelings [35]. As illustrated in Figure 1, digital trace data need to be contextualized with additional data in order to understand the dynamics of change. There has been considerable progress in the development of algorithms in various fields (e.g. text mining [23]), and these algorithms can complement insights obtained via process mining techniques. However, "humans need to interpret what correlations and models mean, i.e. what are their implications for what people are actually doing, feeling, and thinking" [7, p. 18]. Sense-making remains a cognitive process $[22,6]$, which requires inspiration and creativity [9]. Therefore, process mining can be used as an approach complementary to qualitative research approaches, much in the sense of mixed methods [7]. In this vein, process mining can be combined with more interpretive approaches to theory development, such as grounded theory [6]. Furthermore, in order to analyze digital trace data, researchers need to apply a lexicon that provides a "pre-existing set of concepts, constructs, and their implied relationships" [6, p. 4]. A lexicon serves as a theoretical lens through which data are interpreted and related to a scientific discourse. In the following, we will discuss the use of process mining by drawing on the discourse on organizational routines. 


\section{Using Process Mining to Support Theorizing about Change in Organizations}

\subsection{Organizational Routines}

Organizational routines are defined as repetitive and interdependent patterns of actions that involve multiple actors [36]. They can serve as proxies to investigate organizational change [37]. Central to contemporary research is the idea that routines are dynamic and change over time. These dynamics can be triggered by exogenous factors and interventions [38, 5], but they can also occur endogenously when change unfolds from within the organization [39]. To understand these dynamics, routines are commonly explored by means of process theory and researchers primarily draw on qualitative approaches (e.g. observations and interviews) [40]. In light of the increasing digitalization of organizational work [21], additional insights may be gained when routines research utilizes digital trace data and process mining [11, 41].

We see two central motivations to use process mining in organizational routines research. First, process mining draws on traces of repetitive and recognizable actions that were carried out by multiple actors. Process mining allows us to look at performances carried out by specific actors at specific points in time, and hence, they mark the "performative aspects" of a routine [14]. Second, the discourse on organizational routines provides us with a lexicon to explore process change [6]. For example, the distinction between ostensive and performative aspects [36], or the assumption that routines are mindful accomplishments and dynamic in nature [39], allow us to frame the analysis of the data [6].

We illustrate how process mining can support inductive theorizing about endogenous change unfolding over time, and deductive theorizing about exogenous change following punctuated interventions. For each case, we provide suggestions on how the use of process mining can contribute to the discourse. We emphasize, however, that we could also use inductive theorizing to examine exogenous change and deductive theorizing to investigate endogenous change.

\subsection{Supporting Inductive Theorizing About Endogenous-evolutionary Change}

\section{Computationally-Supported Discovery of} Digitized Routines. Organizational routines are ubiquitous, but they are hard to see [42]. Patterns of interdependent actions are distributed across time and space. In order to understand how they are connected and form a coherent stream of activities, researchers are collecting and analyzing extensive amounts of data. Most commonly, empirical work is carried out by means of qualitative methods, including observations, interviews and analyses of archival data [39]. However, more and more work is entangled with digital technologies. When observing "digitized routines" [19], researchers may face additional challenges as the specific activities which are carried out by actors can hardly be observed or reconstructed through interviews. One way to capture the content of such routines is to ask workers to write down the specific actions they take with specific artifacts (e.g. software tools) [43]. While such a strategy can provide new insights about work processes [44, 20], these data are hard to obtain and cover activities within a limited time frame.

We argue that process mining can support theorizing about digitized routines in three respects. First, by drawing on digital trace data in event logs, process mining automatically generates visual information about a routine. This, in turn, facilitates data collection and analysis. Second, digital trace data record activities that were taken by the use of digital artifacts but might be "hidden" to observers or appear trivial and are not explicitly mentioned in interviews. Third, as digital trace data refer to actual records of human actions, process mining can discover activities in a routine that might have been purposefully concealed. Taken together, process mining allows researchers to gain additional views of a routine over time which can guide additional data collection and analysis [6].

Figure 2 depicts a visualization of a purchase order handling routine of a Dutch company. The figure was generated with a process discovery algorithm in Celonis, a commercial process mining application. It is based on an event log-file from the business process intelligence challenge 2019 [45]. The routine starts with the creation of a purchase order item. After the vendor creates an invoice, the goods and the invoice receipts are recorded and the invoice is cleared. This view simplifies the routine, as it captures the most frequent activities and paths. It is, however, useful to gain an initial overview of the most performed activities and the most frequents paths (transitions of activities), covering $75.7 \%$ of overall activities performed and $8.4 \%$ of overall paths. Such a visualization can provide a starting point to understand how a routine is carried out in most of the cases.

Using the same parameters to mine the routine after some time allows us to see if it has changed in significant ways. Additional data could help us understand why this is the case. 


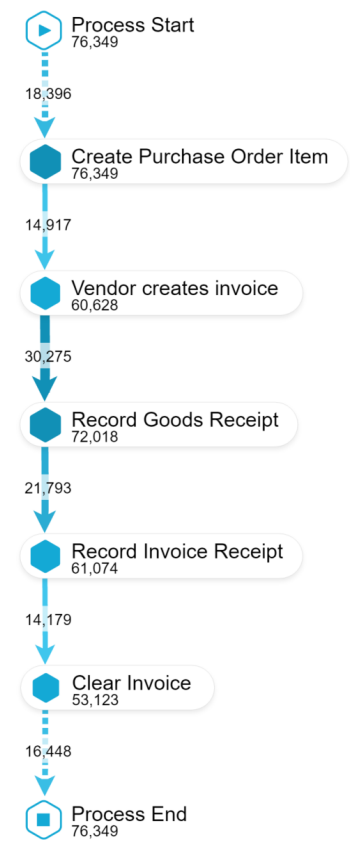

Figure 2. Process Overview

Zooming into Routines and (Emerging) Variations. While routines appear relatively stable and persistent over time, their specific enactments vary. Actors reason, negotiate and make intentional decisions when they execute work [35]. For example, in their ethnographic study on the emergence of routines, Dittrich et al. [39] observed that organizational actors develop variations when they are confronted with unexpected events. Similarly, organizational actors enact different action patterns when work requirements change [46]. While such variations can lead to drifts of whole routines (which we will discuss in the following section), we suggest that process mining can illuminate the many variations existing within a routine, but are only enacted with respect to contextual affordances. A similar argument has been made by Salvato and Rerup [47] who observed that, depending on the goal that is pursued by organizational actors, one routine can have two variations which are relatively stable over time.

Process mining allows us to "zoom into" the routine [24] and detect variations that rarely occur. This can guide subsequent inquiry to understand why variations emerge in relation to contextual factors [19]. Thereby, we may extend existing views on why, how and under what circumstances organizational actors are engaging in alternative enactments of routine work. For example, since digital trace data are equipped with time stamps, we can detect how variations are related to temporal factors (e.g. the presence of a supervisor or shift work)
[18]. Furthermore, since process mining can grant us ad-hoc access to data about organizational work (i.e. while it is being carried out), we are able to explore variations when they occur.

Figure 3 illustrates the idea of how process mining can reveal variations in a routine. We zoom into the purchase order handling routine as depicted in Figure 2 by increasing the path connections to $58.2 \%$, while the relative frequency of activities remains the same. By increasing the granularity, we are able to capture more variations in the routine. We could zoom even further into the routine to reveal additional activities or paths.

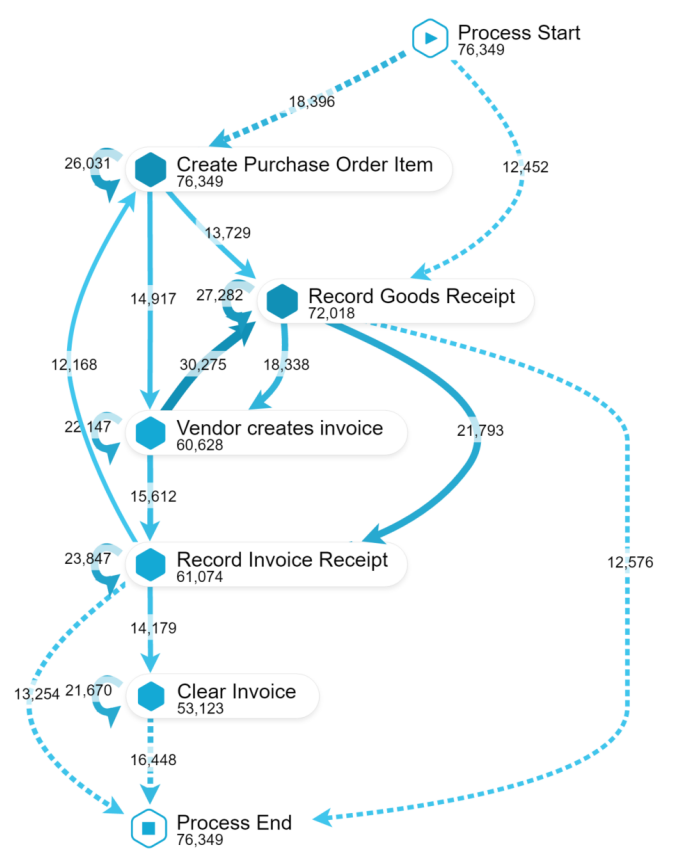

Figure 3. Process Overview

Detecting Microfoundations of Routine Change. Process mining allows us to explore how variations lead to routine change over time. An important characteristic of endogenous change is that it often starts with small incremental changes [2], that is, small variations which are enacted with respect to established themes and previous states. If variations are more likely to be re-enacted, they can become part of the organizational repertoire over time [46]. Organizational routines researchers have a long-standing interest in change dynamics. For example, they have been linked to three actions patterns: selection, retention and variation [16]. However, emerging variations are hard to observe and it may be unclear if and to what extend they are part or become part of a routine.

Process mining can provide a more fine-granular view of patterns of change in routines that are supported 
by digital technologies. Although digital technologies have in-built features that are supposed to facilitate or restrict the enactment of routine activities in certain ways [5], they tend to drift as well [19]. Process mining allows for detecting and visualizing variations that occur along specific actions and are less frequent as compared to how the routine is enacted in the majority of cases.

Changes in the routine can be captured by the use of concept drift algorithms. As explained in section 3.1, these algorithms detect the point in time when the routine changes. For example, the algorithm provided by Maaradji et al. [33] detects significant changes in the sequence of activities as recorded in the event log-file. Once a drift point is identified, the log-file can be separated into sub-logs and a more detailed analysis on each part of the log-file can be done with process discovery algorithms. Thereby, one can draw a more detailed picture of how the routine has changed. For example, activities might be removed or added and paths structures may change such that new dominant paths emerge. Process mining thus allows for discovering "microfoundations" of routine change [48]. By combining these insights with contextual data, we can explore how and why variations become part of a routine.

\subsection{Supporting Deductive Theorizing About Exogenous-punctuated Change}

Most commonly, research on organizational routines follows qualitative-inductive research designs [40]. However, recent approaches treat routines (or aspects of routine work) as dependent variables [38, 18]. In the following, we will illustrate how the use of process mining can contribute to deductive theorizing about routine change and test hypotheses about the enactment of routines with respect to exogenous-punctuated changes over time.

Assessing the Adoption of Artifacts in Routine Enactments. Routines research has been interested in the implementation of artifacts in organizational work [49]. To some extent, the design of an artifact embodies implicit or explicit assumptions about how work should be carried out (e.g. rules) and thus, it intends to guide actions in specific ways [50]. Thus, the expectation of such an implementation is that routines become more efficient and/or effective. However, previous findings highlight that artifacts can disrupt learned patterns of interactions and coordination. They affect work practices in many -often unintended- ways [37]. For example, the implementation of an ERP-system has been linked to the emergence of new action patterns that were not intended by design. By introducing work-arounds, actors bypassed or manipulated the use of the artifact [5]. Recent attempts aim to clarify how artifacts affect enactments of established routines. Drawing on the concept of affordances [51], researchers aim to predict how and under what circumstances actors are more likely to use artifacts. Bapuji et al. [38] conducted a field experiment and found that actors are more likely to adopt an artifact when it facilitates their understandings of how it should be used. In that respect, they identify different affordances. For example, they find that an artifact should facilitate actors' interpretations regarding the actions that they should take. Also, actors should be able to anticipate the outcome when they use a specific artifact.

We can extend such assumptions and use process mining to test the implementation of artifacts with respect to different affordances. For example, if an organization intends to change a routine by means of a new artifact (e.g. new regulations or a digital technology), and if this organization mines the same process in different locations, one could manipulate the artifact implemented in one process and predict how the enactment unfolds in different ways over time, e.g. because certain affordances are incorporated that help actors to develop an understanding about the use of the artifact [38]. Such a field experiment could be performed using a conformance checking algorithm. Hypotheses about the adoption of artifacts could be specified in terms of business process models entailing how the redesigned routine is supposed to be performed. Behavioral measures [34] would then allow to infer how much of the observed behavior is covered in the model, and vice versa. Both routines could then be compared in terms of their respective conformances.

Testing Factors that Influence Change in Routine Enactment. Studies on routines aim to explain how routines change over time. For example, complexity is a measure that can define change in terms of an increasing or decreasing number of possible paths that can be enacted [19]. Recent studies find that complexity changes under different conditions. For example, Pentland et al. [19] report that incremental changes in a routine can increase the number of possible paths and eventually lead to non-linear complexity bursts. After such bursts, the routine takes a new form. Also, it has been suggested that routine variations are dynamic and change in different phases and in relation to work requirements [46].

We can explore a number of hypotheses and test previously made assumptions about routine change against the data we obtain from event logs. For example, we can follow recent propositions and hypothesize that software systems with adaptive programming features 
are less likely to increase complexity in routines [19]. Following the assumption that new organizational actors add variations to a routine [14], we can also make predictions in terms of the number of alternate paths that emerge over time when new workers or customers are involved in the enactment of the routine. We can also investigate the impact of managerial interventions. For example, drawing on recent claims, the enactment of routines has been linked to "retrieval cues" in the organizational environment [52]. When identifying and removing cues associated with certain variations, we could expect that these paths are taken less frequently and are forgotten over time, while at the same time, other paths may become more dominant [19]. This could be examined by analyzing the variants of the routine at different points in time, i.e. before, during and after the intervention.

\section{Discussion}

\subsection{Summary}

We have argued that process mining can provide computational support to theorize about change processes in organizations [6]. While process mining has been developed to analyze and improve business processes in organizations [10], we propose that it can support inductive and deductive theorizing about change in organizations. Process mining uses digital trace data as records of human action [7]. Thereby, it can reveal how processes unfold and change over time. Depending on the size of the event log, process mining can deal with longitudinal data $[1,4,9,11]$. Furthermore, process mining allows us to take a more fine-granular view of process work and visualize actions that might not be recognized by traditional approaches to data collection. Using different algorithms, we can take different perspectives on the data and reveal "critical events" that mark change dynamics [1, 4].

To illustrate our argument, we drew on organizational routines research. Routines research offers a rich lexicon to make sense of the information we gain from process mining $[6,11]$, and we have argued that the use of process mining can complement existing findings about the dynamics of organizational routines. Building on recent claims that call for the use of process mining in routines research [11, 28, 41], we have presented a wider spectrum of algorithms and we have discussed their potential applications in relation to different stages of the research cycle.

Process mining provides an interesting lens to support theorizing on organizational process research, especially as work practices become increasingly entangled with digital technology [21]. Triangulation from different perspectives will advance our understanding of process change and organizational behavior in more general terms, and we believe that process mining adds an exciting new lens to established methodologies.

\subsection{Limitations and Future Research}

We highlighted opportunities for process research that arise from the use of process mining. As pointed out by Berente et al. [6], the use of computationally-driven theorizing is dependent on a lexicon that serves as the theoretical lens through which we explain a certain phenomenon. We drew on the lexicon of organizational routines [36]. This resonates with recent claims in the routines literature. For example, in their recent work on time patterning between and among organizational actors in routines, Turner and Rindova [18, p.2018] suggest that more insights can be gained by combining "fine-grained workflow data with simultaneous surveys or diary accounts". Time-based patterning can be yet another interesting application to use process mining in routines research [18]. However, it is important to note that process theorizing is applied to a plethora of phenomena [3, 12], and process mining can be used in relation to other discourses. For example, it can be used to explore how patterns of actions change while organizations run into crisis and organizational failure [15].

We have argued that process mining can extract processes from digital traces in event logs. Since digital technologies are increasingly entangled with work practices [21] and embedded in organizational routines [5], this approach is useful to make sense of the abundant digital trace data [8]. However, as we have highlighted throughout this article, process mining data only draw a limited picture of a certain phenomenon. To understand how and why a phenomenon unfolds [1], and to identify underlying generative mechanisms [2], we need to account for contextual factors that are not captured in event logs [28].

For illustration purposes, we use the notion of process mining in a general and simplified way. It is important to note that in reality, the use of process mining might not be straight-forward. There are numerous algorithms for discovery, conformance checking and enhancement, and there are also techniques to integrate information about the organizational context [53]. Each algorithm has specific characteristics and leads to different insights (see for example, a recent review of discovery algorithms [29]). In this article, we used an academic license for the 
Celonis process mining application. However, there are many other applications which have different features and may produce different visualizations and analyses. Such aspects are crucial to consider, and the underlying algorithms (and the selected parameters) would need to be made transparent when used in empirical research. Finally, throughout our examples, we assumed that we have access to data that are of high quality. In reality, data gathering and cleaning can be cumbersome processes. These limitations, however, should not discourage future research from using process mining to support theorizing about change in organizations. We hope that this article provides ideas for how process mining can be used with respect to different research questions and designs.

Acknowledgement. The work of Bastian Wurm and Jan Mendling has received funding from the EU H2020 program under the MSCA-RISE agreement 645751 (RISE BPM).

\section{References}

[1] A. H. Van de Ven and G. P. Huber, "Longitudinal field research methods for studying processes of organizational change," Organization science, vol. 1, no. 3, pp. 213-219, 1990.

[2] A. H. van de Ven and M. S. Poole, "Explaining Development and Change in Organizations," The Academy of Management Review, vol. 20, no. 3, pp. 510-540, 1995.

[3] A. Langley, C. Smallman, H. Tsoukas, and A. H. Van de Ven, "Process studies of change in organization and management: Unveiling temporality, activity, and flow," Academy of management journal, vol. 56, no. 1, pp. 1-13, 2013.

[4] A. H. Van de Ven and M. S. Poole, "Alternative approaches for studying organizational change," Organization studies, vol. 26, no. 9, pp. 1377-1404, 2005.

[5] O. Volkoff, D. M. Strong, and M. B. Elmes, "Technological embeddedness and organizational change," Organization science, vol. 18, no. 5, pp. 832-848, 2007.

[6] N. Berente, S. Seidel, and H. Safadi, "Data-Driven Computationally-Intensive Theory Development," Information Systems Research, vol. 30, no. 1, pp. iii-viii, 2019.

[7] A. Lindberg, "Developing Theory through integrating Human \& Machine Pattern Recognition," Forthcoming in Journal of the Association for Information Systems, 2019.

[8] B. Pentland, J. Recker, and I. Kim, "Capturing reality in flight? empirical tools for strong process theory," 2017.

[9] A. Langley, "Strategies for theorizing from process data," Academy of Management review, vol. 24, no. 4, pp. 691-710, 1999.

[10] W. M. P. van der Aalst, A. Adriansyah, and e. a. De Medeiros, Ana Karla Alves, "Process mining manifesto," in Proceedings of the 9th International Conference on Business Process Management (BPM 2011), pp. 169-194, 2011.

[11] B. Pentland, E. Vaast, and J. Ryan Wolf, "Measuring and explaining process dynamics with digital trace data," Unpublished Working Paper, 2019.

[12] A. Langley and H. Tsoukas, "Introducing perspectives on process organization studies," Process, sensemaking, and organizing, vol. 1, no. 9, pp. 1-27, 2010.

[13] T. Hernes and E. Weik, "Organization as process: Drawing a line between endogenous and exogenous views," Scandinavian Journal of Management, vol. 23, no. 3, pp. 251-264, 2007.

[14] B. T. Pentland, T. Hærem, and D. Hillison, "The (n) ever-changing world: Stability and change in organizational routines," Organization Science, vol. 22, no. 6, pp. 1369-1383, 2011.

[15] S. Habersang, J. Küberling-Jost, M. Reihlen, and C. Seckler, "A process perspective on organizational failure: A qualitative meta-analysis," Journal of Management Studies, vol. 56, no. 1, pp. 19-56, 2019.

[16] B. T. Pentland, M. S. Feldman, M. C. Becker, and P. Liu, "Dynamics of organizational routines: A generative model," Journal of Management Studies, vol. 49, no. 8, pp. 1484-1508, 2012.

[17] S. B. Bacharach, P. Bamberger, and W. J. Sonnenstuhl, "The organizational transformation process: The micropolitics of dissonance reduction and the alignment of logics of action," Administrative Science Quarterly, pp. 477-506, 1996.

[18] S. F. Turner and V. P. Rindova, "Watching the clock: Action timing, patterning, and routine performance," Academy of Management Journal, vol. 61, no. 4, pp. 1253-1280, 2018.

[19] B. T. Pentland, P. Liu, W. Kremser, and T. Haerem, "The dynamics of drift in digitized processes," MIS Quarterly, forthcoming.

[20] B. T. Pentland, J. Recker, and G. Wyner, "Rediscovering handoffs," Academy of Management Discoveries, vol. 3, no. 3, pp. 284-301, 2017.

[21] Y. Yoo, O. Henfridsson, and K. Lyytinen, "Research commentary the new organizing logic of digital innovation: an agenda for information systems research," Information systems research, vol. 21, no. 4, pp. 724-735, 2010.

[22] O. Müller, I. Junglas, J. v. Brocke, and S. Debortoli, "Utilizing big data analytics for information systems research: challenges, promises and guidelines," European Journal of Information Systems, vol. 25, no. 4, pp. 289-302, 2016.

[23] T. Schmiedel, O. Müller, and J. vom Brocke, "Topic modeling as a strategy of inquiry in organizational research: A tutorial with an application example on organizational culture," Organizational Research Methods, pp. 941-968, 2018.

[24] J. Gaskin, N. Berente, K. Lyytinen, and Y. Yoo, “Toward Generalizable Sociomaterial Inquiry: A Computational Approach for Zooming In and Out of Sociomaterial Routines," Management Information Systems Quarterly, vol. 38, no. 3, pp. 849-871, 2014.

[25] T. H. Davenport and A. Spanyi, "What Process Mining Is, and Why Companies Should Do It," Harvard Business Review, vol. April, 2019. 
[26] W. M. van Der Aalst, "Process Mining: Overview and Opportunities," ACM Transactions on Management Information Systems, vol. 3, no. 2, p. Article No. 7, 2012.

[27] W. M. P. van der Aalst, Process mining: discovery, conformance and enhancement of business processes. Heidelberg: Springer., 2nd ed., 2011.

[28] B. Wurm, "Patterns of Stability and Change in Business Processes," in Proceedings of the 9th International Workshop on Enterprise Modeling and Information Systems Architectures (EMISA 2018), pp. 21-28, 2018.

[29] A. Augusto, R. Conforti, M. Dumas, M. La Rosa, F. M. Maggi, A. Marrella, M. Mecella, and A. Soo, "Automated discovery of process models from event logs: Review and benchmark," IEEE Transactions on Knowledge and Data Engineering, vol. 31, no. 4, pp. 686-705, 2018.

[30] A. Lindberg, N. Berente, J. Gaskin, and K. Lyytinen, "Coordinating Interdependencies in Online Communities: A Study of an Open Source Software Project," Information Systems Research, vol. 27, no. 4, pp. 751-772, 2016.

[31] B. F. A. Hompes, J. Buijs, W. M. P. van der Aalst, P. M. Dixit, and J. Buurman, "Discovering Deviating Cases and Process Variants Using Trace Clustering," in 27th Benelux Conference on Artificial Intelligence (BNAIC), 2015.

[32] R. P. C. Bose, W. M. Van Der Aalst, I. Žliobaite, and M. Pechenizkiy, "Handling concept drift in process mining," Lecture Notes in Computer Science (including subseries Lecture Notes in Artificial Intelligence and Lecture Notes in Bioinformatics), vol. 6741 LNCS, pp. 391-405, 2011.

[33] A. Maaradji, M. Dumas, M. La Rosa, and A. Ostovar, "Detecting sudden and gradual drifts in business processes from execution traces," IEEE Transactions on Knowledge and Data Engineering, vol. 29, no. 10, pp. 2140-2154, 2017.

[34] A. Polyvyanyy, A. Solti, M. Weidlich, C. D. Ciccio, and J. Mendling, "Monotone precision and recall measures for comparing executions and specifications of dynamic systems," CoRR, vol. abs/1812.07334, 2018.

[35] K. Dittrich and D. Seidl, "Emerging intentionality in routine dynamics: A pragmatist view," Academy of Management Journal, vol. 61, no. 1, pp. 111-138, 2018.

[36] M. S. Feldman and B. T. Pentland, "Reconceptualizing organizational routines as a source of flexibility and change," Administrative science quarterly, vol. 48, no. 1, pp. 94-118, 2003.

[37] L. D'Adderio, "Artifacts at the centre of routines: Performing the material turn in routines theory," Journal of Institutional Economics, vol. 7, no. 2, pp. 197-230, 2011.

[38] H. Bapuji, M. Hora, A. Saeed, and S. Turner, "How understanding-based redesign influences the pattern of actions and effectiveness of routines," Journal of Management, pp. 2132-2162, 2018.

[39] K. Dittrich, S. Guérard, and D. Seidl, "Talking about routines: The role of reflective talk in routine change,' Organization Science, vol. 27, no. 3, pp. 678-697, 2016.

[40] J. Howard-Grenville and C. Rerup, "A process perspective on organizational routines," The SAGE handbook of organization process studies, pp. 323-337, 2016.
[41] D. Breuker and M. Matzner, "Statistical sequence analysis for business process mining and organizational routines," in Proceedings of ECIS 2013, 2013.

[42] B. T. Pentland and M. S. Feldman, "13 issues in empirical field studies of organizational routines 1," Handbook of organizational routines, p. 281, 2008.

[43] B. T. Pentland, "Sequential variety in work processes," Organization Science, vol. 14, no. 5, pp. 528-540, 2003.

[44] B. T. Pentland, "Poetics of process: Finding rhythm and rhyme in processual phenomena using ThreadNet.," 2017.

[45] B. van Dongen, "Dataset bpi challenge 2019. 4tu.centre for research data.." 10.4121/uuid:d06aff4b-79f0-45e6-8ec8-e19730c248f1.

[46] K. Goh and B. T. Pentland, "From actions to paths to patterning: Toward a dynamic theory of patterning in routines," Academy of Management Journal, forthcoming.

[47] C. Salvato and C. Rerup, "Routine regulation: Balancing conflicting goals in organizational routines," Administrative Science Quarterly, vol. 63, no. 1, pp. 170-209, 2018.

[48] B. T. Pentland and T. Hærem, "Organizational routines as patterns of action: Implications for organizational behavior," Annu. Rev. Organ. Psychol. Organ. Behav., vol. 2, no. 1, pp. 465-487, 2015.

[49] B. T. Pentland and M. S. Feldman, "Designing routines: On the folly of designing artifacts, while hoping for patterns of action," Information and organization, vol. 18 , no. 4, pp. 235-250, 2008.

[50] B. T. Pentland and M. S. Feldman, "Narrative networks: Patterns of technology and organization," Organization science, vol. 18, no. 5, pp. 781-795, 2007.

[51] S. Seidel, T. Grisold, and N. Berente, "Modular change in platform ecosystems and routine mirroring in organizations," in Proceedings of the 53rd Hawaii International Conference on System Sciences, forthcoming.

[52] A. Kluge and N. Gronau, "Intentional forgetting in organizations: the importance of eliminating retrieval cues for implementing new routines," Frontiers in psychology, vol. 9, p. 51, 2018.

[53] M. Song and W. M. Van der Aalst, "Towards comprehensive support for organizational mining," Decision Support Systems, vol. 46, no. 1, pp. 300-317, 2008. 